\title{
Suppressive effects of human fetal keratinocytes on the proliferation, differentiation and extracellular matrix synthesis of human hypertrophic scar fibroblasts in vitro
}

\author{
ZHE WANG ${ }^{1}$, QIBIN SONG ${ }^{2}$ and HONGQIU $\mathrm{LI}^{3}$ \\ Departments of ${ }^{1}$ Pathology and ${ }^{2}$ Plastic Surgery, Shengjing Hospital of China Medical University, Shenyang, \\ Liaoning 110004; ${ }^{3}$ Department of Orthopedics, Central Hospital of Shenyang Medical College, \\ Shenyang, Liaoning 110002, P.R. China
}

Received May 29, 2016; Accepted June 8, 2017

DOI: $10.3892 / \mathrm{mmr} .2017 .7220$

\begin{abstract}
A hypertrophic scar is characterized by fibroblast proliferation and excessive extracellular matrix deposition. Emerging evidence has revealed that fetal keratinocytes (KCs) contribute to scarless wound healing. However, the association between fetal keratinocytes and hypertrophic scarring remains unclear. In the present study, human KCs of different gestational ages were isolated and co-cultured with human hypertrophic scar fibroblasts (HSFbs) or normal skin fibroblasts. Gene expression and protein levels of fibronectin, collagen 1and $\alpha$-smooth muscle actin in the fibroblasts were measured by reverse transcription-quantitative polymerase chain reaction and western blot analyses. It was observed that fetal KCs significantly inhibited the proliferation of $\mathrm{HSFb}$ in vitro. Fetal keratinocytes also affected the expression of fibronectin, collagen 1 and $\alpha$-smooth muscle actin in HSFbs. In addition, miR-940 may modulate the suppressive effects of fetal KCs on the cell proliferation, differentiation and extracellular matrix synthesis of HSFbs by directly targeting transforming growth factor- $\beta 1$. Taken together, the results of the present study provide evidence to support the potential use of fetal $\mathrm{KCs}$ for cell-based therapeutic grafting in the prevention of hypertrophic scarring.
\end{abstract}

Correspondence to: Dr Zhe Wang, Department of Pathology, Shengjing Hospital of China Medical University, 36 Sanhao Street, Heping, Shenyang, Liaoning 110004, P.R. China

E-mail:wz_cmu@126.com

Abbreviations: TGF- $\beta 1$, transforming growth factor- $\beta 1$; HS, hypertrophic scar; HSFbs, hypertrophic scar fibroblasts; NFbs, normal skin fibroblasts; ECM, extracellular matrix; KCs, keratinocytes; $\alpha$-SMA, $\alpha$-smooth muscle actin

Key words: fetal keratinocytes, proliferation, hypertrophic scar, collagen, fibroblasts

\section{Introduction}

A hypertrophic scar (HS) is a type of fibrous tissue hyperplasia caused by excessive wound healing following skin and tissue injury. Wound healing in adults usually results in scarring that may cause functional restrictions in movement in addition to physical and psychological difficulties (1-3). Therefore, the development of novel therapeutic approaches to prevent HS formation is necessary. Early- to mid-gestational fetal mammalian skin wounds have been reported to heal rapidly and with the absence of scarring (4-6). The potential of using human fetal skin cells for allogeneic transplantation was recently reported (7). The study demonstrated that fetal keratinocytes (KCs) exhibit shorter expansion times, longer telomeres, reduced immunogenicity and increased clonogenicity, with increased stem cell properties, compared with adult KCs (7). Further previous studies demonstrated that fetal KCs exert important effects on the phenotypic and functional regulation of normal fetal and adult fibroblasts via the secretion of various molecules $(8,9)$. Other studies have demonstrated that early- to mid-gestational fetal KCs may promote scarless fetal wound healing $(6,7)$. However, the association between fetal KCs and HS formation remains unclear.

MicroRNAs (miRNAs/miRs) are a class of small, noncoding RNAs that have roles in skin scarring. miRNAs are between 18 and 25 nucleotides long and alter gene expression by binding to target mRNAs, which subsequently leads to degradation of the target mRNAs or prevents the translation of the target mRNAs into proteins. In a previous study, it was reported that 110 miRNAs, including 22 novel miRNA candidates, were significantly differentially expressed between mid- and late-gestational fetal KCs. A total of 33 differentially expressed miRNAs, including miR-940 and miR-34, were associated with the transforming growth factor- $\beta$ (TGF- $\beta$ ) signaling pathway (10). The effect of miRNAs involved in regulating the TGF- $\beta$ pathway and fibroblast functions has been reported in hypertrophic scarring (11). However, there are few reports regarding the role of miRNAs in fetal $\mathrm{KC}$-induced alterations in human HS fibroblasts (HSFbs).

In the present study, fetal KCs and HSFbs were isolated from the skin tissues of fetuses and adults, respectively, and 
co-cultured using a Transwell system (11). The aim was to determine whether fetal KCs inhibit HS-associated properties of cultured HSFbs, including proliferation, differentiation and extracellular matrix (ECM) synthesis. In addition, the present study investigated the mechanism through which fetal $\mathrm{KCs}$ may inhibit HSFb proliferation, differentiation and ECM synthesis, and determined which factors and mechanisms of prenatal wound healing may be applied to postnatal wound repair.

\section{Materials and methods}

Cell culture. Following approval from the China Medical University Ethics Committee of Shengjing Hospital (Shenyang, China), tissue samples were collected between August 2013 and August 2015 according to the ethical rules for human experimentation stated in the 1975 Declaration of Helsinki. Full-thickness fetal skin tissues were obtained from the lower leg of 5 mid-gestational fetuses ( 3 males and 2 females; gestational age, 20-23 weeks) and 5 late-gestational fetuses ( 3 males and 2 females; gestational age, 30-34 weeks). None of the pregnancies were terminated due to miscarriage or elective abortion, and the reason for termination of pregnancy in each case is summarized in Table I. Women undergoing termination of pregnancy provided written informed consent for the use of fetal tissue in the present study. Samples of HS and paired normal skin tissues were collected during plastic surgery procedures. The HS sample patient profiles are summarized in Table II. All patients provided informed consent. All tissues were collected at the Shengjing Hospital of China Medical University. No patients had been diagnosed with a systemic disease or previously treated for scarring. KCs and $\mathrm{HSFb}$ were cultured as described previously $(8,9,12)$. Cell cultures at passage 3-5 were used in all experiments.

Co-culture of human HSFbs and fetal KCs. For co-culture of HSFbs and fetal KCs, KCs were seeded at a density of $1 \times 10^{5}$ cells $/ \mathrm{cm}^{2}$ on Transwell clear polyester membrane inserts with a $0.4-\mathrm{mm}$ pore size (Costar; Corning Incorporated, Corning, NY, USA) in Epilife ${ }^{\mathrm{TM}}$ growth medium (Invitrogen; Thermo Fisher Scientific, Inc., Waltham, MA, USA) supplemented with $1 \%$ human keratinocyte growth supplement (Invitrogen; Thermo Fisher Scientific, Inc.) and grown to confluence at $37^{\circ} \mathrm{C}, 100 \%$ humidity and $5 \% \mathrm{CO}_{2}$. The medium was subsequently changed to high-glucose Dulbecco's modified Eagle's medium (DMEM; Gibco; Thermo Fisher Scientific, Inc.) with 10\% fetal bovine serum (FBS; Invitrogen; Thermo Fisher Scientific, Inc.), and the cells were raised to the air-liquid interface. Prior to co-culture, HSFbs were seeded at a density of $10^{5}$ cells $/ \mathrm{cm}^{2}$ onto six-well plates in high-glucose DMEM with $10 \% \mathrm{FBS}$, incubated for $48 \mathrm{~h}$ at $37^{\circ} \mathrm{C}$, and subsequently cultured in serum-free medium for an additional $24 \mathrm{~h}$ at $37^{\circ} \mathrm{C}$. Following washing with PBS, the membranes containing $\mathrm{KCs}$ were transferred onto the cultured fibroblasts and maintained at $37^{\circ} \mathrm{C}$, in serum-free low calcium $\mathrm{KC}$ growth medium supplemented with $5 \mathrm{ng} / \mathrm{ml}$ epidermal growth factor for 2 days. HSFbs cultured in isolation with an empty insert served as the control group. All experimental and control samples were produced in triplicate.
miRNA target prediction. TargetScan Human (www. targetscan.org/vert_61) was used to identify the potential targets of miR-940.

Transfection of miRNA mimics and inhibitors into fetal KCs. miR-940 mimics (cat. no. MSY0004983; Qiagen GmbH, Hilden, Germany), miR-940 inhibitors (cat. no. MIN0004983; Qiagen $\mathrm{GmbH}$ ) and a negative control duplex (NC; cat. no. MSY0002505; Qiagen $\mathrm{GmbH}$ ) were employed to investigate the functions of miR-940 in fetal KCs. Fetal KCs $\left(5 \times 10^{5}\right)$ were seeded in six-well plates (Nest Biotechnology Co., Ltd., Wuxi, China). At $70 \%$ confluence, the cells were subjected to $50 \mathrm{mM}$ transfections for $48 \mathrm{~h}$ using Lipofectamine 2000 (Invitrogen; Thermo Fisher Scientific, Inc.), according to the manufacturer's protocol (13).

Cell proliferation assay. Fibroblast proliferation was measured using a tetrazolium reagent, 2-(4-indophenyl)-3-(4-nitrophenyl)-5-(2, 4-disulphophenyl)-2H-tetrazolium monosodium salt (WST-1; cat. no. C0036; Cell Counting kit; Beyotime Institute of Biotechnology, Haimen, China) at $48 \mathrm{~h}$ after the initiation of co-culture, according to the manufacturer's protocol. The optical density was measured by determining the absorbance at $450 \mathrm{~nm}$. The results are expressed as the ratio compared with the control. Data were collected from three independent experiments, each of which was performed in duplicate.

Reverse transcription-quantitative polymerase chain reaction (RT-qPCR) analysis. To detect miRNAs from fetal KCs and fetal epidermis specimens, RNAs were prepared from cells and epidermis using the mirVana miRNA Isolation kit (Ambion; Thermo Fisher Scientific, Inc.). The RT-qPCR for miRNA analysis in fetal KCs was performed using iQ SYBR-Green Supermix (Bio-Rad Laboratories, Inc., Hercules, CA, USA). A total of $3 \mu \mathrm{g}$ total RNA was reverse-transcribed using a specific looped RT primer for each miRNA with a RevertAid First Strand cDNA Synthesis kit (Thermo Fisher Scientific, Inc.). qPCR was performed on an Applied Biosystems 7500 system (Thermo Fisher Scientific, Inc.). U6 was used as an internal control. Total RNA was prepared from fibroblasts at $48 \mathrm{~h}$ after the initiation of co-culture using TRIzol Reagent (Invitrogen; Thermo Fisher Scientific, Inc.), according to the manufacturer's protocol. The fibroblasts were washed three times with PBS prior to extraction. To analyze mRNA expression in fibroblasts, $0.5 \mu \mathrm{g}$ total RNA was reverse-transcribed using random hexamer primers and Moloney murine leukemia virus reverse transcriptase under the conditions recommended by the manufacturer (Invitrogen; Thermo Fisher Scientific, Inc.). The iQ SYBR-Green Supermix and Applied Biosystems 7500 system were additionally used for qPCR of mRNAs in fibroblasts. GAPDH was used as an internal control. Fold changes of miRNA and mRNA expression were calculated using the $2^{-\Delta \Delta C q}$ method (14). The RT-qPCR reaction conditions were $95^{\circ} \mathrm{C}$ for $30 \mathrm{sec}$, followed by 40 cycles of $95^{\circ} \mathrm{C}$ for $5 \mathrm{sec}$ and $60^{\circ} \mathrm{C}$ for $34 \mathrm{sec}$. Primer sequences are presented in Table III.

Dual luciferase reporter assays. Dual luciferase reporter assays were performed as reported previously (15). Fetal mid-gestational KCs were seeded at a density of 5,000 cells/well in 96-well plates and co-transfected with a 
Table I. Reasons for pregnancy termination in each case.

\begin{tabular}{|c|c|c|c|c|}
\hline Case & $\begin{array}{c}\text { Gestationage, } \\
\text { weeks }\end{array}$ & Sex & Ethnicity & Reason \\
\hline 1 & 20 & Male & Chinese & $\begin{array}{l}\text { Congenital } \\
\text { deformities }\end{array}$ \\
\hline 2 & 23 & Male & Chinese & Mother's life at risk \\
\hline 3 & 23 & Male & Chinese & Accidental trauma \\
\hline 4 & 21 & Female & Chinese & Accidental trauma \\
\hline 5 & 23 & Female & Chinese & $\begin{array}{l}\text { Congenital } \\
\text { deformities }\end{array}$ \\
\hline 6 & 31 & Male & Chinese & Mother's life at risk \\
\hline 7 & 30 & Male & Chinese & Accidental trauma \\
\hline 8 & 34 & Male & Chinese & Accidental trauma \\
\hline 9 & 32 & Female & Chinese & $\begin{array}{l}\text { Congenital } \\
\text { deformities }\end{array}$ \\
\hline 10 & 30 & Female & Chinese & Mother's life at risk \\
\hline
\end{tabular}

pMir-Report luciferase vector $(1.2 \mu \mathrm{g})$ containing a wild-type or mutant form of the $3^{\prime}$ untranslated region of TGF- $\beta 1$, which contains a miR-940 binding site, pRL-TK Renilla luciferase vector $(0.12 \mu \mathrm{g}$; Promega Corporation, Madison, WI, USA) and miR-940 mimics $(50 \mathrm{mM})$. At $48 \mathrm{~h}$ post-transfection, the luciferase activities were determined with the Dual-Luciferase Reporter Assay System (Promega Corporation). Renilla luciferase activity was used as an internal control. Following normalization to Renilla luciferase activity, the firefly luciferase activity was analyzed. All results are representative of three independent assays.

Western blot analysis. Total protein was extracted from the indicated cells using a Mammalian Cell Lysis kit (Sigma-Aldrich; Merck KGaA, Darmstadt, Germany) according to the manufacturer's protocol. Cells were washed with ice-cold PBS prior to lysis by mechanical disruption. The protein concentration was determined using the Bradford method. A total of $50 \mu \mathrm{g}$ proteins mixed in loading buffer were separated on $1 \mathrm{~mm}$ NuPage Novex 10\% Bis-Tris gels using a NuPage MOPS SDS Buffer kit (Thermo Fisher Scientific, Inc.) followed by electrotransfer to $0.2-\mathrm{mm}$ nitrocellulose membranes (Pall Life Sciences, Port Washington, WI, USA). Nonspecific binding sites were blocked with $5 \%$ bovine serum albumin (Sigma-Aldrich; Merck KGaA) in PBS for $1 \mathrm{~h}$ at room temperature. Membranes were subsequently incubated with primary antibodies against fibronectin (cat. no. ab23750; diluted 1:500; Abcam, Cambridge, MA, USA), collagen 1 (cat. no. 84336; diluted 1:500; Cell Signaling Technology, Inc., Danvers, MA, USA) or $\alpha$-smooth muscle actin ( $\alpha$-SMA, cat. no. 19245; diluted 1:1,000; Cell Signaling Technology, Inc.) at $4^{\circ} \mathrm{C}$ overnight. Following three washes with PBS containing $0.5 \%$ Tween-20, the membranes were incubated with a horseradish peroxidase-conjugated secondary antibody (cat. no. NA934; diluted 1:10,000; GE Healthcare Life Sciences, Little Chalfont, UK) at room temperature for $2 \mathrm{~h}$. Signals were visualized with Amersham Enhanced Chemiluminescence Prime Western Blotting Detection Reagent (GE Healthcare Life Sciences). For the protein loading control, blots were stripped
Table II. Patient profiles for donors of HSFbs and NFbs.

\begin{tabular}{llcl}
\hline No. & Sex & Age, years & Biopsy site \\
\hline 1 & Male & 32 & Leg \\
2 & Female & 24 & Shoulder \\
3 & Female & 27 & Leg \\
4 & Male & 35 & Leg \\
5 & Female & 24 & Neck \\
6 & Male & 34 & Leg \\
7 & Female & 33 & Leg \\
8 & Female & 28 & Leg \\
\hline
\end{tabular}

Collected normal skin was $5 \mathrm{~cm}$ from the hypertrophic scar tissue. HSFbs, hypertrophic scarring fibroblasts; NFbs, normal skin fibroblasts.

and stained for GAPDH using an anti-GAPDH antibody (cat. no. ab8245; diluted 1:2,000; Abcam).

TGF- $\beta 1$ rescue experiments. TGF- $\beta 1$ rescue experiments were performed by the addition of $5 \mathrm{ng} / \mathrm{ml}$ human recombinant active TGF- $\beta 1$ (hraTGF- $\beta 1$; PeproTech EC Ltd., London, UK) to cultures of $10^{6} \mathrm{KCs}$ that had been transfected with miR-940 mimcs at $37^{\circ} \mathrm{C}$ for $24 \mathrm{~h}$. Fetal KCs were harvested at $48 \mathrm{~h}$ post-transfection. Cell proliferation and TGF- $\beta 1$ expression were analyzed as described above with a cell proliferation assay and western blotting, respectively.

Statistical analysis. Data are presented as the mean \pm standard deviation. Comparisons between two groups were performed using Student's t-test. Multiple intervention experiments were compared by one-way analysis of variance with Tukey's correction for multiple pairwise comparisons. Statistical analysis was performed using GraphPad Prism 6 software (GraphPad Software Inc., La Jolla, CA, USA). P<0.05 was considered to indicate a statistically significant difference.

\section{Results}

Characterization of HSFbs. HSFbs and normal skin fibroblasts (NFbs) were isolated from HS and normal tissues, respectively. In order to compare fibroblasts from HS and normal tissues, cell proliferation and $\alpha$-SMA expression were examined in HSFbs and NFbs. As presented in Fig. 1A, HSFbs maintained notably increased proliferation rates relative to $\mathrm{NFbs}$ on days 3-6. RT-qPCR demonstrated significantly increased expression of $\alpha$-SMA mRNA in HSFbs compared with NFbs at passages 4 and 5 (Fig. 1B).

Fetal KCs inhibit proliferation, differentiationand ECM synthesis in HSFbs. Primary $\mathrm{KCs}$ were isolated from fetal skin tissue and healthy adult skin tissue. HSFbs were isolated from HS tissue. In order to determine whether fetal epidermal KCs exerted any biological effects on $\mathrm{HSFb}$ proliferation, a co-culture model of $\mathrm{KCs}$ and fibroblasts was employed (Fig. 2). Quantitative analysis demonstrated that fetal mid-gestational KCs exhibited a more potent suppressive effect on the proliferation of HSFbs at 2 days 
Table III. Primers for reverse transcription-quantitative polymerase chain reaction analysis.

Gene

Forward primer

Reverse primer

Amplicon

length, bp

\begin{tabular}{llll}
\hline Collagen 1 & CCCGGGTTTCAGAGACAACTTC & TCCACATGCTTTATTCCAGCAATC & 150 \\
Fibronectin & GAGTTGTCGTGGTCCCTCAG & TGGAGGCGGCATCATAGTTG & 119 \\
$\alpha$-SMA & TCCACTTGGAAGTTGGCTTCA & TGTCCGTGTAGTCCTCCCTG & 144 \\
GAPDH & TGTGGGCATCAATGGATTTGG & ACACCATGTATTCCGGGTCAAT & 116 \\
\hline
\end{tabular}

A

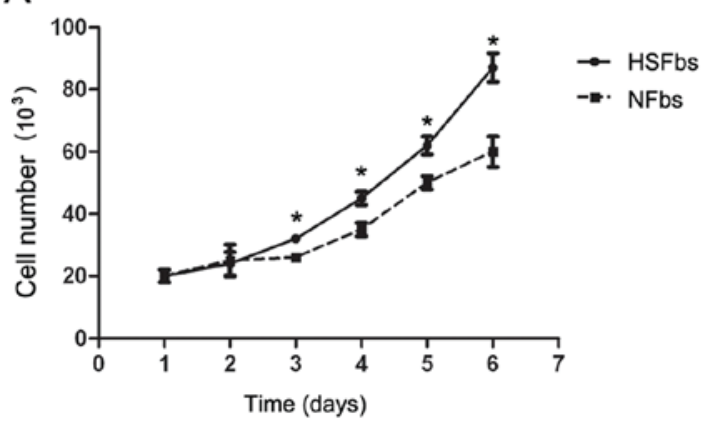

B

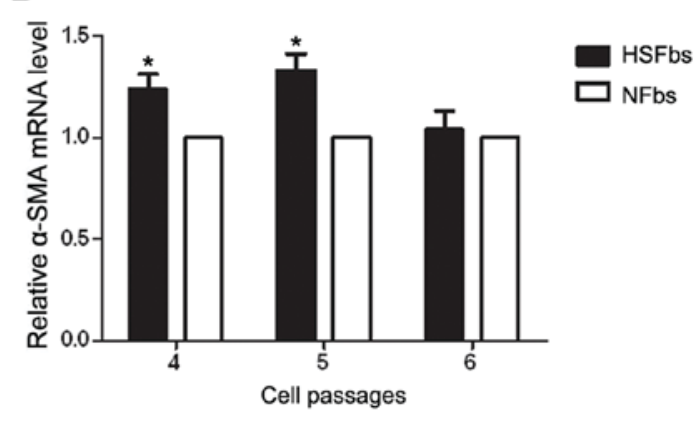

Figure 1. Characterization of HSFbs. (A) Growth curves of HSFbs and NFbs at passage 5. There was a significant difference in cell proliferation between HSFbs and NFbs on days 3-6 (P<0.05). (B) Representative reverse transcription-quantitative polymerase chain reaction analysis of $\alpha$-SMA expression at passages 4,5 and 6 . The graph illustrates corresponding densitometric analysis of $\alpha$-SMA normalized to GAPDH for each passage, $n=3$. ${ }^{*} \mathrm{P}<0.05$ vs. $\mathrm{NFbs}$. HSFbs, hypertrophic scar fibroblasts; NFbs, normal skin fibroblasts; $\alpha$-SMA, $\alpha$-smooth muscle actin.

of culture, compared with late-gestational or adult $\mathrm{KCs}$ (Fig. 2A). Fibronectin and collagen are major ECM components. Therefore, the expression of fibronectin and collagen 1 in HSFbs co-cultured with KCs was detected. The data demonstrated that fetal mid-gestational KCs exhibited a more potent suppressive effect on ECM synthesis in HSFbs, compared with late-gestational or adult KCs (Fig. 2B-D). In order to determine whether fetal epidermal KCs exerted any biological effects on HSFb differentiation, $\alpha$-SMA expression was examined in HSFbs co-cultured with fetal and adult KCs. As presented in Fig. 2B and E, fetal mid-gestational KCs exhibited a more potent suppressive effect on the expression of $\alpha$-SMA in HSFbs, compared with late-gestational or adult KCs.

miR-940 is upregulated in fetal KCs. In order to investigate the exact mechanism through which fetal KCs inhibit $\mathrm{HSFb}$ proliferation, differentiation and ECM synthesis, nextgeneration sequencing was performed in our previous study to measure the expression of miRNA in fetal KCs. It was observed that 110 miRNAs, including 22 novel miRNA candidates, were significantly differentially expressed between mid- and late-gestational fetal KCs (10). Experimental assessment of candidate miRNAs in the present study demonstrated that miR-940 was upregulated in fetal mid-gestational KCs (Fig. 3A). The levels of miR-940 were also detected in 5 human fetal mid-gestational epidermal specimens, 5 late-gestational epidermal specimens and 5 adult epidermal specimens by RT-qPCR. The results demonstrated that miR-940 was upregulated in mid-gestational epidermal specimens (Fig. 3B).
Role of miR-940 in the suppressive effects of fetal mid-gestational KCs on HSFb proliferation, differentiation and ECM synthesis. In order to assess the role of miR-940 in the suppressive effects of fetal $\mathrm{KCs}$ on $\mathrm{HSFb}$ proliferation, cell proliferation was examined in HSFbs co-cultured with fetal $\mathrm{KCs}$, in the presence or absence of miR-940 mimics, miR-940 inhibitors or NC. As presented in Fig. 4A and B, miR-940 expression was confirmed by RT-qPCR. The results of the proliferation analysis demonstrated that transfection of miR-940 mimics enhanced cell proliferation inhibition induced by fetal mid-gestational $\mathrm{KCs}$, whereas transfection of miR-940 inhibitors reduced cell proliferation inhibition induced by fetal mid-gestational $\mathrm{KCs}$ $(\mathrm{P}<0.05$; Fig. 4C). In order to assess the role of miR-940 in the suppressive effects of fetal KCs on the expression of fibronectin, collagen 1 and $\alpha$-SMA in HSFbs, RT-qPCR and western blot analyses were performed in HSFbs co-cultured with fetal KCs, in the presence or absence of miR-940 mimics, miR-940 inhibitors or NC. The data demonstrated that transfection of miR-940 mimics enhanced the inhibition offibronectin, collagen 1 and $\alpha$-SMA expression in HSFbs induced by fetal mid-gestational $\mathrm{KCs}$, whereas transfection of miR-940 inhibitors led to an upregulation of fibronectin, collagen 1 and $\alpha$-SMA expression in HSFbs, compared with the NC group (Fig. 4D-G). The results of the present study indicated that miR-940 may act as a positive modulator in the suppressive effects of fetal KCs on HS formation.

miR-940 directly targets TGF- $\beta 1$ in KCs. To obtain additional insight into the molecular mechanisms of miR-940 in the suppressive effects of fetal KCs on cell proliferation, differentiation, and ECM synthesis of HSFbs, the public miRNA 

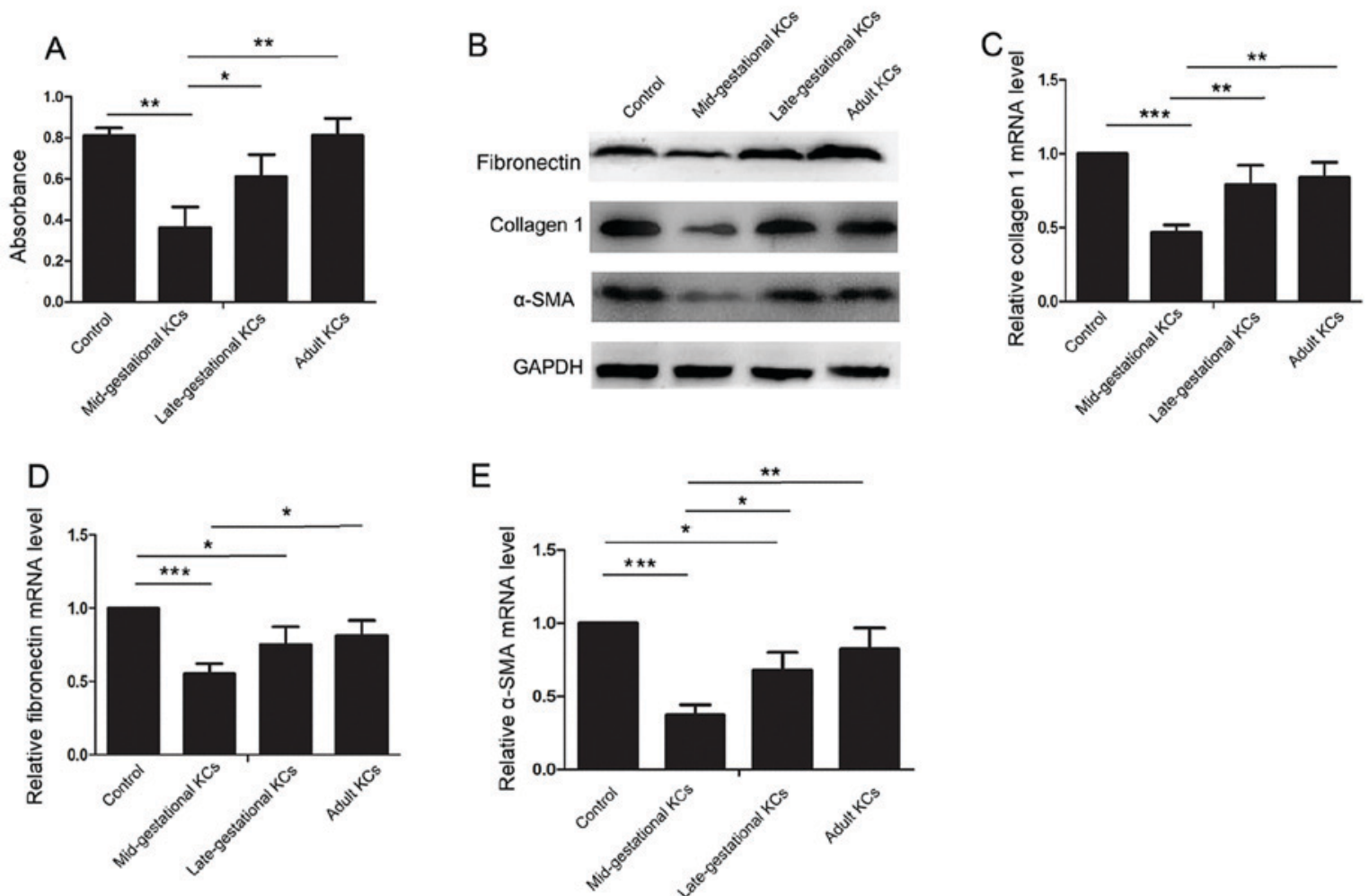

Figure 2. Effect of fetal KCs on the proliferation, differentiation and extracellular matrix synthesis of HSFbs. (A) At $48 \mathrm{~h}$ subsequent to the beginning of co-culture, cell proliferation was measured. HSFbs co-cultured with fetal KCs for $48 \mathrm{~h}$ demonstrated a reduced proliferation capacity compared with control HSFbs. There was a significant difference in HSFb proliferation between mid- and late-gestational or adult groups at $48 \mathrm{~h}$ subsequent to the beginning of co-culture. (B) The levels of fibronectin, collagen 1 and $\alpha$-SMA proteins were determined by western blotting at $48 \mathrm{~h}$ subsequent to the beginning of co-culture. GAPDH was used as a loading control. The levels of (C) collagen 1, (D) fibronectin and (E) $\alpha$-SMA mRNA were determined by reverse transcription-quantitative polymerase chain reaction analysis. There was a significant difference in the mRNA levels of fibronectin, collagen 1 and $\alpha$-SMA between mid- and late-gestational or adult groups at $48 \mathrm{~h}$ subsequent to the beginning of co-culture. Each value is expressed as the mean \pm standard deviation of three independent experiments, $n=5 .{ }^{*} \mathrm{P}<0.05,{ }^{* *} \mathrm{P}<0.01$ and ${ }^{* * * *} \mathrm{P}<0.001$, as indicated. KCs, keratinocytes; HSFbs, hypertrophic scar fibroblasts; $\alpha$-SMA, $\alpha$-smooth muscle actin.

A

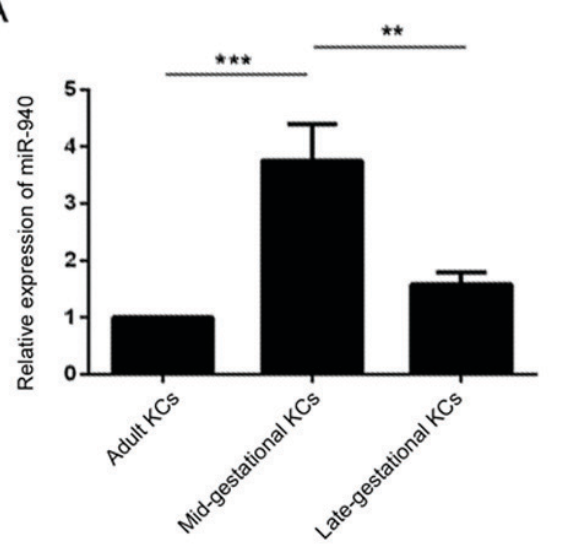

B

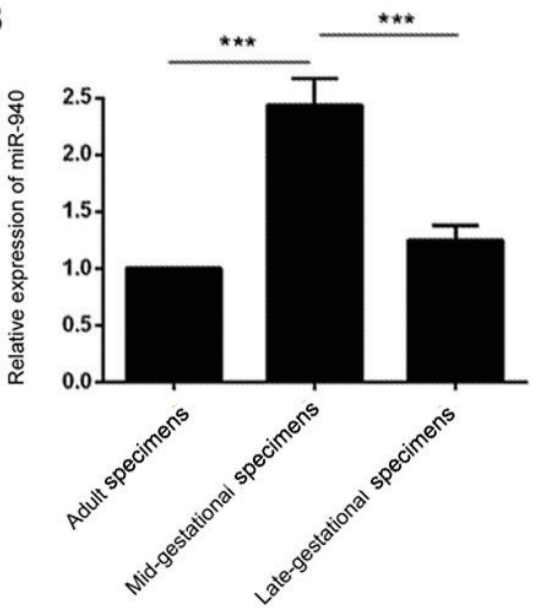

Figure 3. High expression of miR-940 in human fetal KCs and fetal epidermal tissues. (A) miR-940 was upregulated in human fetal KCs. There was a significant difference in the levels of miR-940 between mid-and late-gestational or adult groups. (B) miR-940 was upregulated in human fetal epidermis specimens. There was a significant difference in the levels of miR-940 between mid- and late-gestational or adult groups. ${ }^{* *} \mathrm{P}<0.01$ and ${ }^{* * *} \mathrm{P}<0.001$, as indicated. miR, microRNA; KCs, keratinocytes.

database (www. targetscan.org/vert_61) was used to predict the potential targets of miR-940. Due to a highly-conserved binding site, TGF- $\beta 1$ was selected for further examination. In order to determine the association between miR-940 and
TGF- $\beta 1$, the protein expression of TGF- $\beta 1$ was measured in fetal mid-gestational KCs with differential expression of miR-940. TGF- $\beta 1$ protein levels in fetal mid-gestational KCs were markedly increased following downregulation of 

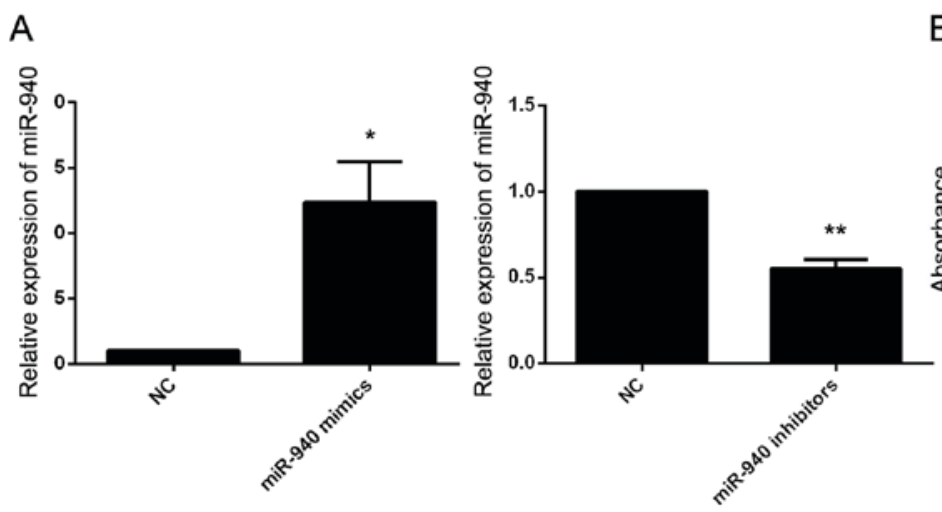

C

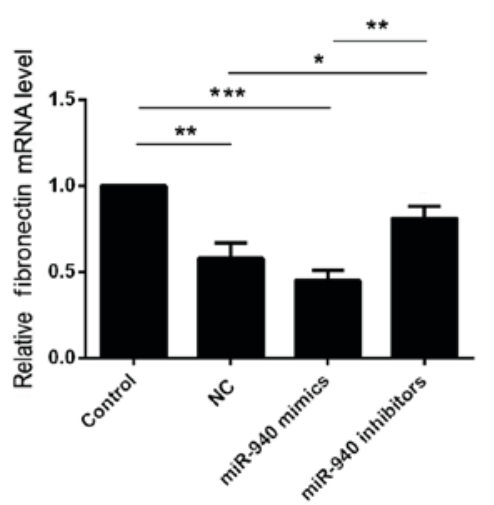

$\mathrm{E}$

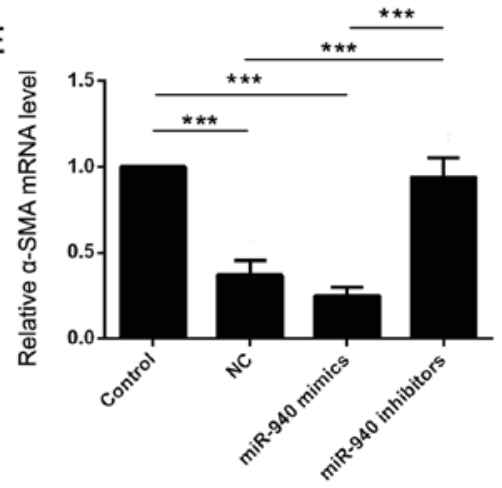

B

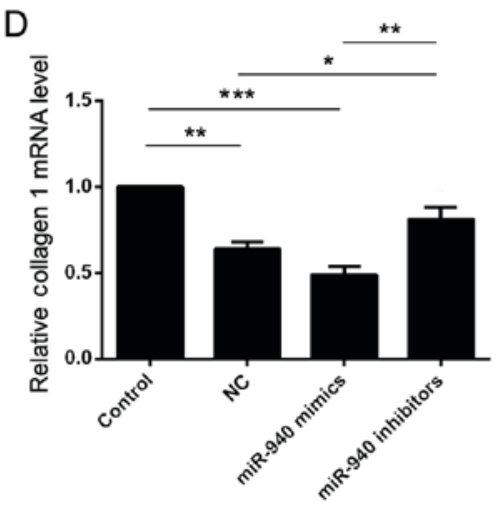

$\mathrm{F}$

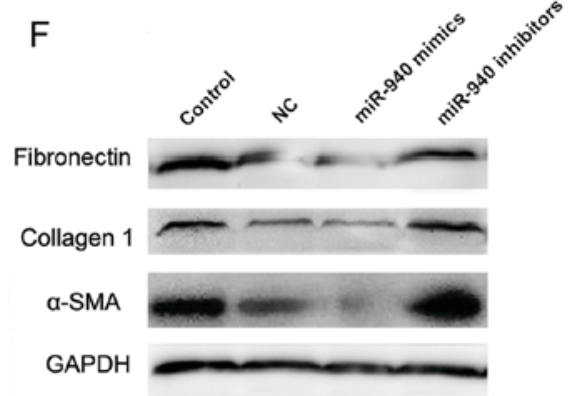

Figure 4. Role of miR-940 in the suppressive effects of fetal mid-gestational KCs on HSFb proliferation, differentiation and extracellular matrix synthesis. (A) Fetal mid-gestational KCs were transfected withan NC duplex, miR-940 mimics or miR-940 inhibitors, and the miR-940 expression levels were analyzed using RT-qPCR. (B) Cell proliferation assays demonstrated the role of miR-940 in the suppressive effects of fetal mid-gestational KCs on HSFb proliferation. The levels of (C) fibronectin, (D) collagen 1 and (E) $\alpha$-SMA mRNA in HSFbs were determined by RT-qPCR at $48 \mathrm{~h}$ subsequent to the beginning of co-culture. (F) The levels of fibronectin, collagen 1 and $\alpha$-SMA proteins in HSFbs were determined by western blotting at $48 \mathrm{~h}$ subsequent to the beginning of co-culture GAPDH was used as a loading control. Each value is expressed as the mean \pm standard deviation of three independent experiments, $\mathrm{n}=5$. HSFbs cultured in isolation with an empty insert served as the control group. ${ }^{*} \mathrm{P}<0.05,{ }^{* *} \mathrm{P}<0.01$ and ${ }^{* * *} \mathrm{P}<0.001$, as indicated. miR, microRNA; KCs, keratinocytes; HSFbs, hypertrophic scar fibroblasts; NC, negative control; RT-qPCR, reverse transcription-quantitative polymerase chain reaction; $\alpha$-SMA, $\alpha$-smooth muscle actin.

miR-940 (Fig. 5A). To confirm whether TGF- $\beta 1$ is a direct target of miR-940, dual luciferase reporter vectors containing the putative TGF- $\beta 1$ 3'-untranslated region target site for miR-940, which was downstream of the luciferase gene (pMir-TGF- $\beta 1$-wild-type), and a deletion mutant of $5 \mathrm{bp}$ in the seed region (pMir-TGF- $\beta 1$-mutant), were constructed. As presented in Fig. 5B, luciferase activity assays demonstrated that miR-940 significantly reducedthe activity of wild-type, although not mutant, reporters in fetal mid-gestational KCs. The results of the present study demonstrated the specificity of miR-940 in targeting TGF- $\beta 1$.

TGF- $\beta 1$ is involved in the effects of fetal KCs on cell proliferation, differentiation and ECM synthesis in HSFbs. The present study investigated whether the effect of miR-940 overexpression on the suppressive effects of fetal KCs on cell proliferation, differentiation and ECM synthesis of HSFbs was mediated by TGF- $\beta 1$. TGF- $\beta 1$ rescue experiments were performed by the addition of $5 \mathrm{ng} / \mathrm{ml}$ hraTGF- $\beta 1$ to cultures of KCs that had been transfected with miR-940 mimics. As presented in Fig. 6A, the proliferation of HSFbs co-cultured with KCs was assessed by WST- 1 assays. TGF- $\beta 1$ notably decreased the suppressive effects of human fetal KCs on the cell proliferation of HSFbs. In addition, fibronectin, collagen 1 and $\alpha$-SMA expression in HSFbs co-cultured with $\mathrm{KCs}$ in the presence of $5 \mathrm{ng} / \mathrm{ml}$ hraTGF- $\beta 1$ was examined by RT-qPCR analysis and western blotting. TGF- $\beta 1$ reduced the suppressive effects of human fetal mid-gestational KCs on the expression 
A

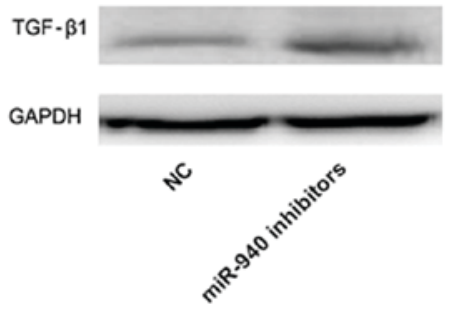

$\mathrm{B}$

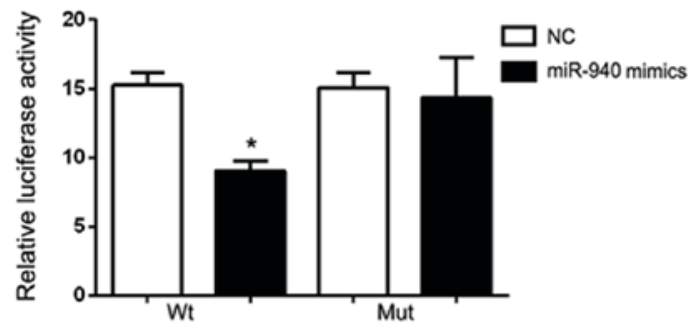

Figure 5. miR-940 directly targets TGF- $\beta 1$ in fetal KCs. (A) Western blotting of TGF- $\beta 1$ in fetal mid-gestational KCs transfected with NC or miR-940 inhibitors. (B) Luciferase activities of the pMir-TGF- $\beta 1-3$ '-untranslated region reporter in fetal mid-gestational KCs co-transfected with pRL-TK Renilla luciferase vector and miR-940 mimics. KCs transfected with the NC duplex served as the control. P-values were derived from at least three independent experiments."P<0.05 vs. NC. miR, microRNA; TGF- $\beta 1$, transforming growth factor- $\beta 1$; KCs, keratinocytes; NC, negative control; Wt, wild-type; Mut, mutant.

A

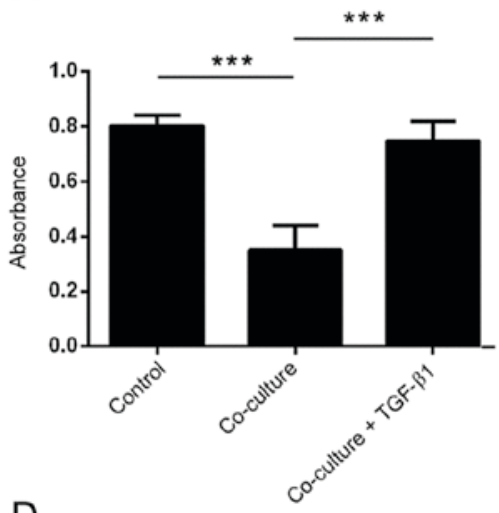

D

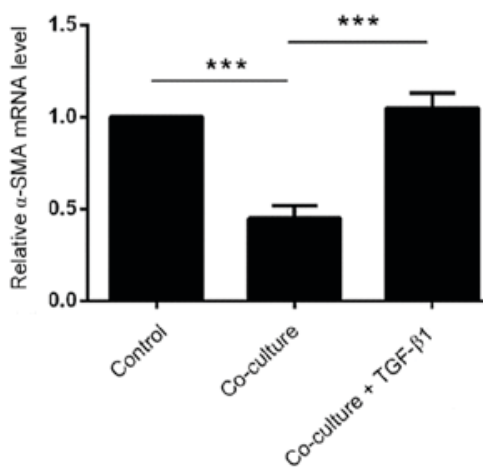

B

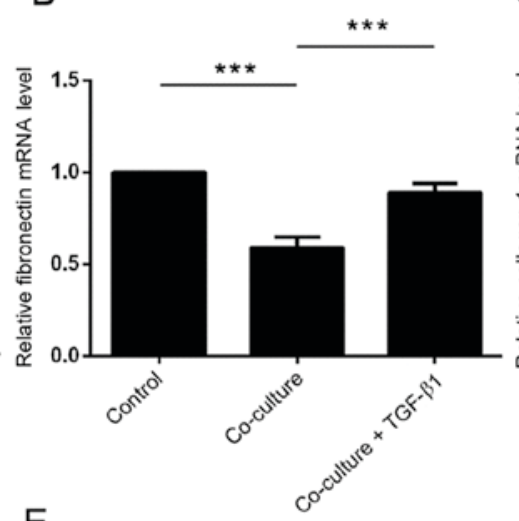

E

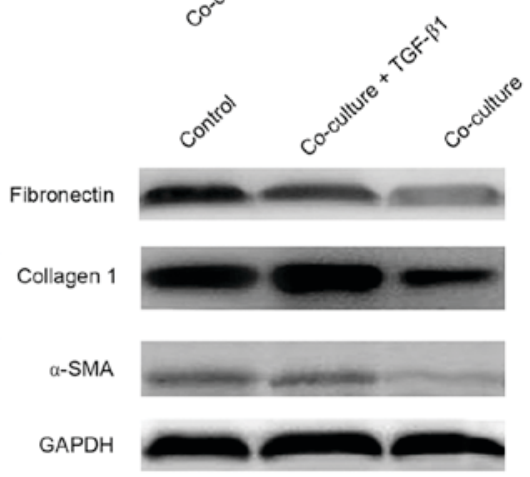

C

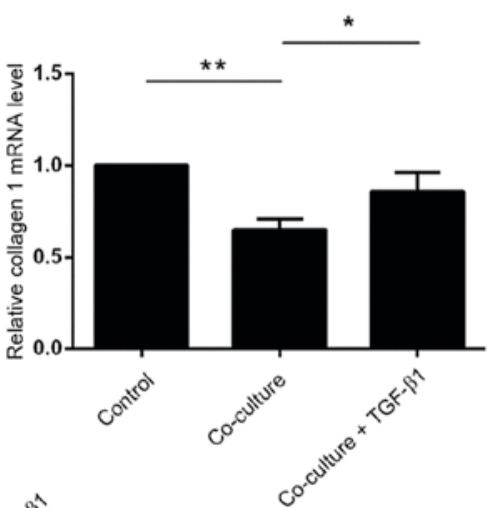

Figure 6. Expression of TGF- $\beta 1$ reduces the suppressive effects of fetal mid-gestational keratinocytes overexpressing microRNA-940 on HSFb proliferation, differentiation and extracellular matrix synthesis. (A) Cell proliferation assays demonstrated differences in the growth of the various groups. The levels of (B) fibronectin, (C) collagen 1 and (D) $\alpha$-SMA mRNA in HSFbs were determined by reverse transcription-quantitative polymerase chain reaction analysis at $48 \mathrm{~h}$ subsequent to the beginning of co-culture. (E) The levels of fibronectin, collagen 1 and $\alpha$-SMA proteins in HSFbs were determined by western blotting at $48 \mathrm{~h}$ subsequent to the beginning of co-culture. GAPDH was used as a loading control. Each value is expressed as the mean \pm standard deviation of three independent experiments, $\mathrm{n}=5$. HSFbs cultured in isolation with an empty insert served as the control group. ${ }^{*} \mathrm{P}<0.05,{ }^{* *} \mathrm{P}<0.01$ and ${ }^{* * *} \mathrm{P}<0.001$, as indicated. TGF- $\beta 1$, transforming growth factor- $\beta 1$; HSFbs, hypertrophic scar fibroblasts; $\alpha$-SMA, $\alpha$-smooth muscle actin.

of fibronectin, collagen 1 and $\alpha$-SMA (Fig. 6B-E). These results revealed the involvement of TGF- $\beta 1$ in the suppressive effect of fetal KCs on the proliferation, differentiation and ECM synthesis of HSFbs.

The present study demonstrated that miR-940 mimic transfection enhances fetal mid-gestational KC-mediated suppression of cell proliferation, differentiation and ECM synthesis of HSFbs, at least partially via downregulation of TGF- $\beta 1$ expression (Fig. 7).

\section{Discussion}

HS formation is a complex and multifactorial fibrotic abnormality that is associated with excessive fibroblast proliferation and ECM deposition $(1,16,17)$. Current therapeutic strategies for this condition are inadequate $(16,18)$. Grafting of cultured KCs to promote regeneration was one of the first clinical applications for cell therapy (19). Researchers have observed that early- to mid-gestational fetal KCs promote fetal 


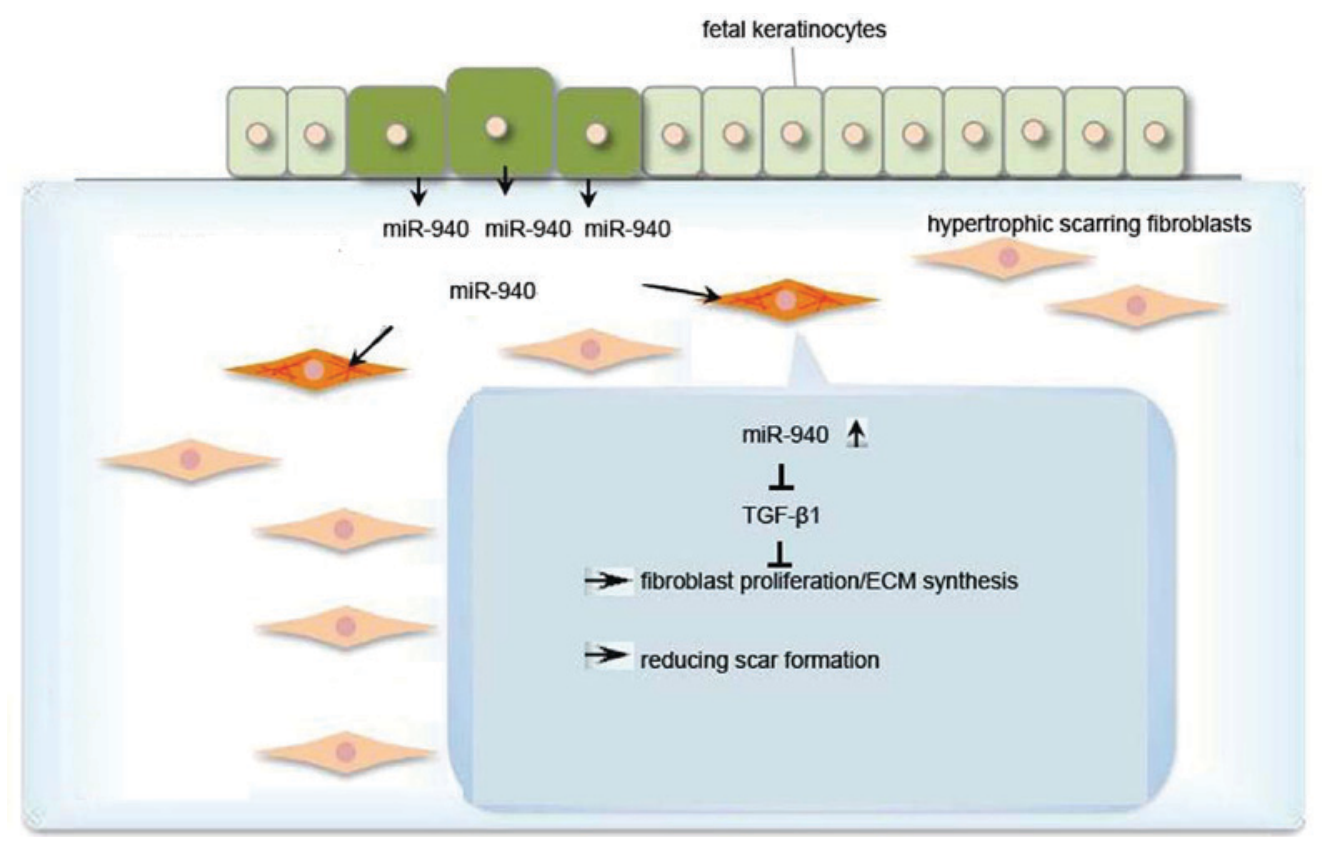

Figure 7. Schematic representation summarizing the roles of miR-940 and TGF- $\beta 1$ in the inhibitory effects of fetal mid-gestational keratinocytes on hypertrophic scar fibroblast proliferation, differentiation and ECM synthesis. miR, microRNA; TGF- $\beta 1$, transforming growth factor $\beta 1$; ECM, extracellular matrix.

scarless wound healing (20). Previous studies demonstrated that mid-gestational KCs exhibited positive effect on proliferation, migration, collagen synthesis and ECM remodeling in fibroblasts from 20-30-year-olds (8,9). However, the underlying mechanisms, and whether fetal KCs may be effective in the prevention of HS formation, are yet to be established. The results of the present study indicated that fetal KCs, particularly mid-gestational KCs, decrease fibroblast proliferation, differentiation and ECM synthesis in HSFbs. Fetal mid-gestational $\mathrm{KC}$ grafting may represent a potential therapeutic strategy for the prevention or treatment of HS.

Excessive collagen synthesis is regarded as a pathological characteristic of HS formation (21-25). In the present study, it was observed that fetal KCs reduced themRNA and protein expression of collagen 1 in HSFbs. In addition to collagen 1, fibronectin is an important component of the ECM andis upregulated in HS formation. In the present study, it was observed that fetal KCs significantly decreased the mRNA and protein expression of fibronectin in HSFbs. In addition, a significant difference in the levels of fibronectin and collagen 1 mRNA expression was observed between mid- and late-gestational or adult groups at $48 \mathrm{~h}$ after the beginning of co-culture. The results of the present study indicated that mid-gestational KCs inhibit ECM synthesis in HSFbs.

During the active period of HS formation, fibroblasts differentiate into myofibroblasts, which exhibit a high proliferative capacity and synthesize large quantities of ECM molecules. $\alpha$-SMA is an established marker of myofibroblasts. Additionally, HS formation causes irreversible tissue contracture and contributes to the release of TGF- $\beta 1$, which subsequently leads to the continuous stimulation of myofibroblast differentiation and activity (26). The present study demonstrated that fetal mid-gestational KCs decreased the expression of $\alpha$-SMA mRNA and protein in HSFbs, which was accompanied by inhibition of cell proliferation and ECM synthesis. A significant difference was also observed in the levels of $\alpha$-SMA mRNA expression between mid- and late-gestational or adult groups, at $48 \mathrm{~h}$ after the beginning of co-culture. The results of the present study indicated that fetal mid-gestational KCs may inhibit $\mathrm{HSFb}$ differentiation.

Gene therapy based on miRNA has been proposed as a novel therapeutic approach (27). The role of miRNAs in skin development and pathology has been extensively researched. Several previous reports have indicated the involvement of miRNAs in the regulation of HS formation $(11,28-30)$. However, limited information regarding the roles of miRNAs in fetal KC-induced alterations in HSFbs is available (31). In order to clarify which miRNAs are involved in the suppressive effect of fetal KCs on HS formation, particularly the regulation of $\mathrm{HSFb}$ proliferation, it was previously demonstrated that 110 miRNAs, including 22 novel miRNA candidates, were significantly upregulated in fetal KCs; 33 differentially expressed miRNAs and miR-34 family members are associated with the TGF- $\beta$ pathway (10). Experimental assessment of candidate miRNAs in the present study demonstrated that miR-940 was highly upregulated in fetal mid-gestational KCs. These results indicated a role for miR-940 in the regulation of $\mathrm{HSFb}$ proliferation, differentiation and ECM synthesis. The present study aimed to clarify the biological role of miR-940 in HSFb proliferation, differentiation, and ECM synthesis regulation by fetal mid-gestational KCs. Downregulation of miR-940 by transient transfection with miR-940 inhibitors or upregulation of the target gene (TGF- $\beta 1$ ) blocked the suppressive effects of fetal mid-gestational $\mathrm{KCs}$ on proliferation, differentiation and ECM synthesis in HSFbs.

In conclusion, the results of the present study indicated that fetal KCs may regulate $\mathrm{HSFb}$ proliferation, differentiation and ECM synthesis. Specifically, the present study revealed that miR-940 mimic transfection enhances fetal mid-gestational 
KC-mediated suppression of cell proliferation, differentiation and ECM synthesis in HSFbs, at least partially via downregulation of TGF- $\beta 1$ expression. The present results support the hypothesisthat the suppressive effects of fetal KCs on the proliferation of HSFbs are partially mediated by an increase in miR-940 expression. Whether similar effects may be observed in clinical situations involving HS formation remains to be determined. If similar effects are involved in clinical situations, modification of fetal KCs by miRNAs may provide a novel therapeutic strategy to prevent HS formation. Further research is required to completely elucidate the suppressive effects on HS of fetal KCs.

\section{Acknowledgements}

The present study was supported by the National Natural Science Foundation (grant no. 81601692) and the Program of Shengjing Hospital (grant no. MD53).

\section{References}

1. Kwan P, Ding J and Tredget EE: MicroRNA 181b regulates decorin production by dermal fibroblasts and may be a potential therapy for hypertrophic scar. PLoS One 10: e0123054, 2015.

2. Khan MA, Bashir MM and Khan FA: Intralesional triamcinolone alone and in combination with 5-fluorouracil for the treatment of keloid and hypertrophic scars. J Pak Med Assoc 64: 1003-1007, 2014.

3. Xue M and Jackson CJ: Extracellular matrix reorganization during wound healing and its impact on abnormal scarring. Adv Wound Care (New Rochelle) 4: 119-136, 2015.

4. Walmsley GG, Maan ZN, Wong VW, Duscher D, Hu MS, Zielins ER, Wearda T, Muhonen E, McArdle A, Tevlin R, et al: Scarless wound healing: Chasing the holy grail. Plast Reconstr Surg 135: 907-917, 2015 .

5. Walmsley GG, Hu MS, Hong WX, Maan ZN, Lorenz HP and Longaker MT: A mouse fetal skin model of scarless wound repair. J Vis Exp 16: 52297, 2015.

6. Manuel J and Gawronska-Kozak B: Matrix metalloproteinase 9 (MMP-9) is upregulated during scarless wound healing in athymic nude mice. Matrix Biol 25: 505-514, 2006.

7. Tan KK, Salgado G, Connolly JE, Chan JK and Lane EB: Characterization of fetalkeratinocytes, showing enhanced stem cell-like properties: A potential source of cells for skin reconstruction. Stem Cell Reports 3: 324-338, 2014.

8. Wang Z, Liu X, Zhang D, Wang X, Zhao F, Zhang T, Wang R, Lin X, Shi P and Pang X: Phenotypic and functional modulation of 20-30 year old dermal fibroblasts by mid-and late-gestational keratinocytes in vitro. Burns 41: 1064-1075, 2015.

9. Wang Z, Liu X, Zhang D, Wang X, Zhao F, Shi P and Pang X: Co-culture with human fetal epidermal keratinocytes promotes proliferation and migration of human fetal and adult dermal fibroblasts. Mol Med Rep 11: 1105-1110, 2015.

10. Zhao F, Wang Z, Lang H, Liu X, Zhang D, Wang X, Zhang T, Wang R, Shi P and Pang X: Dynamic expression of novel MiRNA candidates and MiRNA-34 family members in early- to mid-gestational fetal keratinocytes contributes to scarless wound healing by targeting the tgf- $\beta$ pathway. PLoS One 10: e0126087, 2015.

11. Xiao K, Luo X, Wang X and Gao Z: MicroRNA-185 regulates transforming growth factor- $\beta 1$ and collagen- 1 in hypertrophic scar fibroblasts. Mol Med Rep 15: 1489-1496, 2017.

12. Li P, He QY and Luo CQ: Overexpression of miR-200b inhibits the cell proliferation and promotes apoptosis of human hypertrophic scar fibroblasts in vitro. J Dermatol 41: 903-911, 2014.
13. Ye Q, Wang SJ, Chen JY, Rahman K, Xin HL and Zhang H: Medicinal plants for the treatment of hypertrophic scars. Evid Based Complement Alternat Med 2015: 101340, 2015.

14. Livak KJ and Schmittgen TD: Analysis of relative gene expression data using real-time quantitative PCR and the 2(-Delta Delta C(T)) method. Methods 25: 402-408, 2001.

15. Clément T, Salone V and Rederstorff M: Dual luciferase gene reporter assays to study miRNA function. Methods Mol Biol 1296: 187-198, 2015.

16. Tredget EE, Levi B and Donelan MB: Biology and principles of scar management and burn reconstruction. Surg Clin North Am 94: 793-815, 2014.

17. Green H: The birth of therapy with cultured cells. Bioessays 30 : 897-903, 2008

18. Gold MH, McGuire M, Mustoe TA, Pusic A, Sachdev M, Waibel J and Murcia C; International Advisory Panel on Scar Management: Updated international clinical recommendations on scar management: Part 2-algorithms for scar prevention and treatment. Dermatol Surg 40: 825-831, 2014.

19. Babalola O, Mamalis A, Lev-Tov H and Jagdeo J. Optical coherence tomography (OCT) of collagen in normal skin and skin fibrosis. Arch Dermatol Res 306: 1-9, 2014.

20. Mast BA, Diegelmann RF, Krummel TM and Cohen IK: Scarless wound healing in the mammalian fetus. Surg Gynecol Obstet 174: 441-451, 1992.

21. Atiyeh BS: Nonsurgical management of hypertrophic scars: Evidence-based therapies, standard practices and emerging methods. Aesthetic Plast Surg 31: 468-494, 2007.

22. Bláha J: Physiology and pathology of skin after burns and derangement of gene expression. Acta Chir Plast 48: 127-132, 2006.

23. Al-Qattan MM, Abd-Elwahed MM, Hawary K, Arafah MM and Shier MK: Myofibroblast expression in skin wounds is enhanced by collagen III suppression. Biomed Res Int 2015: $958695,2015$.

24. Zhu H, Luo H and Zuo X: MicroRNAs: Their involvement in fibrosis pathogenesis and use as diagnostic biomarkers in scleroderma. Exp Mol Med 45: e41, 2013.

25. Gras C, Ratuszny D, Hadamitzky C, Zhang H, Blasczyk R and Figueiredo C: miR-145 Contributes to hypertrophic scarring of the skin by inducing myofibroblast activity. Mol Med 21: 296-304, 2015.

26. Jinnin M: Recent progress in studies of miRNA and skin diseases. J Dermatol 42: 551-558, 2015.

27. Liu Y, Yang D, Xiao Z and Zhang M: miRNA expression profiles in keloid tissue and corresponding normal skin tissue. Aesthetic Plast Surg 36: 193-201, 2012.

28. Ren Y, Deng CL, Wan WD, Zheng JH, Mao GY and Yang SL: Suppressive effects of induced pluripotent stem cell-conditioned medium on in vitro hypertrophic scarring fibroblast activation. Mol Med Rep 11: 2471-2476, 2015.

29. Liu X, Wang Z, Wang R, Zhao F, Shi P, Jiang Y and Pang X: Direct comparison of the potency of human mesenchymal stem cells derived from amnion tissue, bone marrow and adipose tissue at inducing dermal fibroblast responses to cutaneous wounds. Int J Mol Med 31: 407-415, 2013.

30. Zhang D, Wang J, Wang Z, Zhang T, Shi P, Wang X, Zhao F, Liu X, Lin $X$ and Pang X: miR-136 modulates TGF- $\beta 1$-induced proliferation arrest by targeting PPP2R 2A in keratinocytes. Biomed Res Int 2015: 453518, 2015.

31. Wang Z, Li H, Zhang D, Liu X, Zhao F, Pang X and Wang Q: Effect of advanced glycosylation end products on apoptosis in human adipose tissue-derived stem cells in vitro. Cell Biosci 5: 3,2015 . 